\title{
A COMPOSITION THEOREM FOR GENERAL UNITARY TRANSFORMS ${ }^{1}$
}

\author{
CHARLES FOX
}

1. General Unitary Transforms are of the form

$$
\begin{aligned}
& \int_{0}^{\infty}(k(a, y))^{*} f(y) d y=\int_{0}^{\alpha} g(x) d x, \\
& \int_{0}^{\infty}(l(a, y))^{*} g(y) d y=\int_{0}^{\alpha} f(x) d x,
\end{aligned}
$$

where $(k(a, y))^{*}$ denotes the conjugate complex of $k(a, y)$, with an analogous interpretation elsewhere whenever a function is enclosed by ( )*. Together with (1) and (2) we also have the Parseval formula as follows: writing (1) in the operational form $T f(y)=g(x)$ and supposing that we also have $T f_{1}(y)=g_{1}(x)$ then

$$
\int_{0}^{\infty} f(y)\left(f_{1}(y)\right)^{*} d y=\int_{0}^{\infty} g(x)\left(g_{1}(x)\right)^{*} d x .
$$

We shall refer to $k(a, x) ; l(a, x)$ as a pair of unitary kernels.

Bochner [1] proves that $k(a, x) ; l(a, x),(a \geqq 0)$ form a pair of unitary kernels if and only if condition (A) below holds and equations (4), (5) and (6) are all satisfied: (A) considered as functions of $x$, $k(a, x) \in L^{2}(0, \infty), l(a, x) \in L^{2}(0, \infty)$,

$$
\begin{aligned}
\int_{c}^{\infty} k(a, x)(k(b, x))^{*} d x & =\min (a, b), \\
\int_{0}^{\infty} l(a, x)(l(b, x))^{*} d x & =\min (a, b), \\
\int_{0}^{b}(k(a, x))^{*} d x & =\int_{0}^{a} l(b, x) d x .
\end{aligned}
$$

If (A), (4), (5) and (6) are satisfied and if in (1) $f(x) \in L^{2}(0, \infty)$ then we also have $g(x) \in L^{2}(0, \infty)$. Detailed proofs can be found in Bochner and Chandrasekharan [2, Chap. 5].

I prove the following theorem: Let $k(a, x) ; l(a, x)$ be one pair of

Presented to the Society April 5, 1957; received by the editors February 7, 1957.

1 This research was carried out with the support of a Summer Research Associateship of the National Research Council of Canada. 
unitary kernels and $p(a, x) ; q(a, x)$ be another pair of unitary kernels. Then the functions $m(a, x) ; n(a, x)$ defined in (7) and (8) below are also a pair of unitary kernels.

$$
\begin{aligned}
& m(a, x)=\frac{\partial}{\partial x} \int_{0}^{\infty}(k(x, y))^{*} p(a, y) d y, \\
& n(a, x)=\frac{\partial}{\partial x} \int_{0}^{\infty} k(a, y)(p(x, y))^{*} d y .
\end{aligned}
$$

Evidently, if we know two pairs of unitary kernels we can, by means of this result, find a large number of other pairs.

To prove the theorem we must establish (A), (4), (5) and (6) with $k(a, x)$ and $l(a, x)$ replaced by $m(a, x)$ and $n(a, x)$ respectively. Integrating (7) with respect to $x$ from 0 to $b$ we have

$$
\int_{0}^{\infty}(k(b, y))^{*} p(a, y) d y=\int_{0}^{b} m(a, x) d x .
$$

We now look upon (9) as part of a general unitary transform such as (1) with kernel $k(b, y), f(y)=p(a, y)$ and $g(x)=m(a, x)$. Since $p(a, x)$ is a kernel we have, from (A), $p(a, x) \in L^{2}(0, \infty)$ and therefore $m(a, x) \in L^{2}(0, \infty)$, both regarded as functions of $x$.

Again, from (3), we have

$$
\int_{0}^{\infty} m(a, x)(m(b, x))^{*} d x=\int_{0}^{\infty} p(a, y)(p(b, y)) * d y .
$$

Since $p(a, x)$ is a kernel it must satisfy an equation analogous to (4) and therefore

$$
\int_{0}^{\infty} m(a, x)(m(b, x)) * d x=\min (a, b) .
$$

If (8) is integrated with respect to $x$ from 0 to $b$ and the result is regarded as a general unitary transform with kernel $p(b, y)$, we can prove similarly that $n(a, x) \in L^{2}(0, \infty)$ (considered as a function of $x$ ) and that

$$
\begin{aligned}
\int_{0}^{\infty} n(a, x)(n(b, x))^{*} d x & =\int_{0}^{\infty} k(a, y)(k(b, y))^{*} d y \\
& =\min (a, b)
\end{aligned}
$$

from (4).

Finally from (8) we see that 


$$
\begin{aligned}
\int_{0}^{a}(n(b, x)) * d x & =\int_{0}^{\infty}(k(b, y)) * p(a, y) d y \\
& =\int_{0}^{b} m(a, x) d x
\end{aligned}
$$

from (9). This completes the proof, since we have now established (A), (4), (5) and (6) with $k(a, x)$ and $l(a, x)$ replaced by $m(a, x)$ and $n(a, x)$ respectively.

2. In [1] Bochner introduces two operators $U$ and $V$, by means of which he obtains relations between step functions and pairs of unitary kernels. These operators can also be used to express the relationships between the functions $k(a, x), p(a, x), m(a, x)$ and $n(a, x)$.

If $g_{a}$ denotes the step function $g_{a}=1 ; 0<x<a, g_{a}=0 ; a \leqq x$, then the operators $U$ and $V$ are defined as follows:

$$
U g_{a}=k(a, x) ; \quad V g_{a}=l(a, x) .
$$

With the usual notation for inner product, namely

$$
(f, g)=\int_{0}^{\infty} f(g)^{*} d x
$$

we also have, from (6),

$$
\left(g_{b}, U g_{a}\right)=\left(V g_{b}, g_{a}\right) .
$$

It is proved in [1] that these operators are inverse to each other and in $[2$, Theorem 78$]$ that (18) can be extended so as to apply to all functions in $L^{2}(0, \infty)$ as well as to step functions.

Now, with $U$ and $V$ as defined by (16) let us define $U^{\prime}$ and $V^{\prime}$ by

$$
U^{\prime} g_{a}=p(a, x) ; \quad V^{\prime} g_{a}=q(a, x) .
$$

We have

$$
\begin{aligned}
\left(m(a, x), g_{b}\right) & =\int_{0}^{b} m(a, x) d x \\
& =\int_{0}^{\infty}(k(b, y))^{*} p(a, y) d y
\end{aligned}
$$

from (9)

$$
=\left(U^{\prime} g_{a}, U g_{b}\right)
$$

from (17) 


$$
=\left(V U^{\prime} g_{a}, g_{b}\right)
$$

from the extension of (18). Since $b$ is arbitrary, it follows that $m(a, x)$ $=V U^{\prime} g_{a}$ almost everywhere. In a similar manner, starting from (8), we can also prove that $n(a, x)=V^{\prime} U g_{a}$ almost everywhere.

\section{REFERENCES}

1. S. Bochner, Inversion formulae and unitary transforms, Ann. of Math. vol. 55 (1934) pp. 111-115.

2. S. Bochner and K. Chandrasekharan, Fourier transforms, Princeton University Press.

McGill University

\section{A PROPERTY OF THE LAPLACE TRANSFORMATION}

P. G. ROONEY

1. Introduction. While certain of the properties of the Laplace transformation are so well known that they have become engineering tools, there are others that have received very little attention, and yet are very interesting. One of these comes about as follows. Let $f(s)$ be the Laplace transform of $\phi(t)$, that is

I

$$
f(s)=\int_{0}^{\infty} e^{-s t} \phi(t) d t=\mathfrak{L}(\phi(t) ; s) .
$$

Then under certain conditions,

II

$$
\mathcal{L}\left(\phi\left(t^{2}\right) ; s\right)=\frac{s}{4 \pi^{1 / 2}} \int_{0}^{\infty} e^{-y} y^{-3 / 2} f\left(\frac{s^{2}}{4 y}\right) d y ;
$$

this formula is given, for example, in $[2,4.1(22)]$. At least one generalization of this formula is known, that giving $\mathscr{L}\left(t^{\nu} \phi\left(t^{2}\right) ; s\right)$-see $[2,4.1(22)$ and $(23)]$ - but we propose to generalize here in a different direction, namely that of replacing the $y^{-3 / 2}$ in the right-hand integral of II by $y^{\nu-1}$. Specifically, we propose to show that, under certain conditions 1957.

Received by the editors November 29, 1956 and, in revised form, February 20, 